\title{
Time-dependent stellar-mass binary black hole mergers in AGN disks: Mass distribution of hierarchical mergers
}

\author{
Guo-Peng $\mathrm{Li}^{1}{ }^{1, *}$ \\ ${ }^{1}$ Laboratory for Relativistic Astrophysics, Department of Physics, Guangxi University, \\ Nanning 530004, China
}

(Dated: March 9, 2022)

\begin{abstract}
There is much debate about the channels for astrophysical origins of the stellar-mass binary black hole (BBH) mergers detected by LIGO and Virgo. Active galactic nuclei (AGNs) are promising sites for the efficient formation and rapid mergers of $\mathrm{BBHs}$ due to migration traps in high-density gas disks within an inner radii. In this paper, we carry out Monte Carlo simulations to explore the mass properties of mergers over time and hierarchical mergers-with one of the black holes (BHs) being the remnant of a previous merger. We find that the predicted merger rate is $\sim 27-37 \mathrm{Gpc}^{-3} \mathrm{yr}^{-1}$ and the detection rate of LIGO/Virgo accompanying with extreme mass-ratio mergers will increase in the late stage of AGNs. The fraction of hierarchical mergers is $\sim 24 \%$, and its mass-ratio peak is $\sim 0.15-0.35$. Compared with the low-generation mergers in hierarchical mergers, the mass ratio of high-generation mergers has a flatter distribution around its peak. These reveal that the $\mathrm{BBH}$ mergers detected by LIGO/Virgo with the extreme mass ratio or heavy component masses can be well explained in the AGN channel.
\end{abstract}

\section{INTRODUCTION}

There are more than 80 stellar-mass binary black hole (BBH) mergers detected by Advanced LIGO [1] and Advanced Virgo [2]. However, there is much debate about the astrophysical formation path to mergers. The two main channels to form compact binary mergers are (1) isolated binary evolution [3-6] accompanied by mass transfer [7], commonenvelope ejection $[8,9]$, envelope expansion [10], or chemically homogeneous evolution in a tidally distorted binary [11, 12]; (2) dynamical formation [13] in young star clusters [14-16], globular clusters [17, 18], nuclear star clusters [1922], or active galactic nucleus (AGN) disks [23-28]. In addition, there are some alternative scenarios for mergers, such as primordial black hole (PBH) binary mergers $[29,30]$ and triples or few-body configurations [31,32].

Compared with other formation channels, the $\mathrm{BBH}$ mergers in the AGN disk have the following properties. (1) The mass distribution of black holes (BHs) which participate in mergers is heavier than other channels due to the hardening of AGN disks [33]. This can explain the heavy component masses of BBHs detected by LIGO/Virgo. (2) Component masses in the "mass gap" or a extreme mass ratio become general via hierarchical BBH mergers (see a review by Ref. [34]). It is shown that in the migration traps, $\mathrm{BHs}$ will merge repeatedly with the remnant of a merger of other two BHs with a high probability $[35,36]$. (3) The BBH mergers may accompany with electromagnetic/neutrino signature owing to a Bondi explosion triggered by hyper-Eddington accretion onto the $\mathrm{BBH}$ [37, 38], ram-pressure stripping of gas within the BH Hill sphere caused by a merger kick [39], or high energy neutrinos from choked gamma-ray bursts in AGN disks [40].

Galactic nuclei are the densest environments of stars and compact objects in the universe [41]. In the active phase of galactic nuclei, a high-density gas disk can efficiently assist

\footnotetext{
*lgp@st.gxu.edu.cn
}

binary formation [26, 35, 42] and mergers [24]. There are tens of thousands of stellar-mass black holes harboring within the innermost parsec in the nuclei of active galaxies due to mass segregation $[43,44]$. These $\mathrm{BHs}$ will interact with the AGN accretion disk which helps align their orbits with the AGN disk [33]. Once the BHs align with the AGN disk, interaction between the gas should move them to migration traps within the disk [23]. Generally, the location of migration traps is about 300 Schwarzschild radii from the central supermassive black hole (SMBH) [35, 42]. Reference [45] proposed that the existing last migration trap within the AGN disk, to about a few Schwarzschild radii of SMBH, such that the mass of a LIGO/Virgo BBH will be higher than the real mass due to doppler and gravitational redshifts. During the migration to migration traps, a binary will be formed by the two BHs if the following two conditions are satisfied. First, the separation of the two BHs is smaller than a mutual Hill radii, $R_{\mathrm{mH}}=\left[\left(m_{i}+m_{j}\right) / 3 M_{\bullet}\right]^{1 / 3}\left[\left(r_{i}+r_{j}\right) / 2\right]$, where $m_{i}$ and $m_{j}$ are the masses of the two BHs and $r_{i}$ and $r_{j}$ are their distances from the SMBH with mass $M_{\bullet}$. Second, the BBH is hard, i.e., the relative kinetic energy of the BBH, $k_{\text {rel }}=1 / 2 \mu v_{\text {rel }}^{2}$, is less than the binding energy, $U=G m_{i} m_{j} /\left(2 R_{\mathrm{mH}}\right)$, where $G$ is the gravitational constant, and $\mu$ and $v_{\text {rel }}$ are the reduced mass of the binary and the relative velocity between the two BHs, respectively. The binaries are also formed with a higher chance in migration traps [26]. Once a BBH is formed, a merger will rapidly occur due to tidal and viscous angular momentum exchange between the binary and the AGN disk [24].

Most of the previous studies focused on migration or migration traps in AGN disks [24, 26, 27, 42]. However, we suggest that orbital alignment of $\mathrm{BHs}$ with respect to the disk is more important than migration and migration traps. Because typical orbital alignment times with the AGN disk are much longer than the time of migration and mergers for $\mathrm{BHs}$ [46], and the BHs aligning with the disk would be heavier [33] and numerous (see more details in Sec. III A) in the early stage of AGNs. These indicate that properties of BBH mergers are different over time. Therefore, in this paper, we consider time-dependence for mergers by dividing five epochs in 
the AGN lifetime, that also can naturally produce a hierarchical merger population. We investigate mass properties taking into account the effects of the time-dependent and the hierarchical mergers, and compute the resulting merger masses and mass ratios.

The rest of this paper is organized as follows. In Sec. II we describe our analytical framework to calculate the formation and mergers of BBHs in AGNs. In Sec. III we present our findings and discuss their implications. We summarize our conclusions in Sec. IV.

\section{METHOD}

We carry out Monte Carlo simulations to obtain the mass distributions of $\mathrm{BBH}$ mergers in AGN disks.

\section{A. Stellar-mass black hole population}

We adopt an initial $\mathrm{BH}$ mass distribution in the galactic nuclei as

$$
\frac{\mathrm{d} N}{\mathrm{~d} m_{\mathrm{bh}}} \propto m_{\mathrm{bh}}^{-\beta} .
$$

The range of $\mathrm{BH}$ masses $m_{\mathrm{bh}} \in\left[5 M_{\odot}, 50 M_{\odot}\right]$ is adopted by considering the lower and pair-instability mass gap. In our fiducial model, we adopt $\beta=2.35$ [33]. It is also indicated that the $\beta$-dependence of results of simulations is weak for $\beta$ in the range $\sim 2-3$ [33].

The mass distribution of BHs changes with the distance from the SMBH $[47,48]$. In general, it is assumed that the semimajor axis $a$ of the orbit of initial BHs from the SMBH obeys $[49,50]$

$$
\frac{\mathrm{d} n}{\mathrm{~d} a} \propto a^{-3 / 2-0.5 m_{\mathrm{bh}} / m_{\max }},
$$

where $m_{\max }=50 M_{\odot}$. The maximal semimajor axis $R_{\text {inf }}=$ $1.2\left(M_{\bullet} / 10^{6} M_{\odot}\right)^{1 / 2}$ pc [51] and the minimal semimajor axis $R_{\text {min }} \sim 10^{-4}$ pc [52] are adopted. For simplicity, we assume $\mathrm{BH}$ orbiting the $\mathrm{SMBH}$ on circular orbits at inclination angle $\psi$ with $\cos \psi$ being uniform in $[-1,1]$ [24]. The number of BHs with the spherical distribution around the $\mathrm{SMBH}$ is $~$ $(1-4) \times 10^{4}[53]$. We use an initial BH component consisted of $\sim 2 \times 10^{4}$ BHs following Refs. [26, 52]. We assume that $\mathrm{BHs}$ are initially in binaries with a certain fraction $f_{\mathrm{b}}$. Here, we follow Ref. [52] to assume $f_{\mathrm{b}}=0.15$ due to soft-binarysingle interactions and deal with the initial binaries using the method of Ref. [24]. We adopt the SMBH mass $M_{\bullet}=10^{6} M_{\odot}$ in our simulations.

\section{B. Orbital alignment, migration, and merger}

The BHs that orbit the SMBH at inclination angle $\psi$ with respect to the AGN disk will align with the disk due to attenuation of their perpendicular velocity $v_{\mathrm{z}}$ with respect to the disk. This is mainly because the BHs will gravitationally capture the gas from the AGN disk every time they cross it. The mass of the captured gas during each crossing is [24, 33]:

$$
\Delta M_{\text {cross }}=\Delta v t_{\text {cross }} r_{\mathrm{BHL}}^{2} \pi \Sigma /(2 H)
$$

where $\Delta v, t_{\text {cross }}$, and $r_{\mathrm{BHL}}$ are the relative velocity of the gas and the $\mathrm{BH}$ upon crossing, the duration of the crossing, and the BH's Bondi-Hoyle-Lyttleton radii, respectively. With $v_{\text {orb }}=\left(G M_{\bullet} / r\right)^{1 / 2}$ and $t_{\text {orb }}=2 \pi r^{3 / 2}\left(G M_{\bullet}\right)^{-1 / 2}$ being the orbital velocity and the orbital period of the BH with mass $m_{\mathrm{bh}}$, one can have

$$
\begin{gathered}
\Delta v=v_{\mathrm{orb}}\left[(1-\cos \psi)^{2}+\sin ^{2} \psi\right]^{1 / 2}, \\
t_{\mathrm{cross}} \approx 2 H /\left(v_{\mathrm{orb}} \sin \psi\right), \\
r_{\mathrm{BHL}}=2 G m_{\mathrm{bh}} /\left(\Delta v^{2}+c_{\mathrm{s}}^{2}\right) .
\end{gathered}
$$

Due to the capture of the gas, the perpendicular velocity $v_{\mathrm{Z}}$ of the $\mathrm{BH}$ will change during each crossing:

$$
\frac{\Delta v_{\mathrm{z}}}{v_{\mathrm{z}}}=\frac{\Delta M_{\mathrm{cross}}}{m_{\mathrm{bh}}},
$$

where $v_{\mathrm{z}}=v_{\text {orb }} \sin \psi$. Therefore, the timescale of orbital alignment with the disk can be approximated as

$$
\tau_{\text {align }} \sim \frac{t_{\text {orb }} v_{\mathrm{z}}}{2 \Delta v_{\mathrm{z}}}
$$

The BHs will move to the migration traps in the AGN disk analogous to protoplanetary migration due to a torque which is caused by the exchange of angular momentum between the gas disk and them [23]. The torque of the BHs will change sign from positive to negative in migration traps, that makes BHs gather together $[35,42]$. It is worthwhile to point out that comparing to the alignment time, the migration time and the merger time are much shorter. Reference [23] showed that a characteristic time of migration is $10^{5} \mathrm{yr}$. Reference [46] also found that the migration time of a binary with masses of $30 M_{\odot}-30 M_{\odot}$ is about $10^{5} \mathrm{yr}$ (see also Ref. [24]). In addition, Ref. [42] showed that the merger time is far less than the migration time in the AGN disk. Therefore, we neglect the time of the migration and mergers in our Monte Carlo simulations.

As a result, $\mathrm{BBH}$ formation and mergers between two $\mathrm{BHs}$ are equivalent in our simulations [42, 54]. In Refs. [42, 54], the authors assumed that the gas hardening prescription [55] holds all the way to the GW regime. This is probably unrealistic, since it is likely that gas hardening becomes less efficient at small separations (or large binary orbital velocities around its center of mass) either due to feedback or lower gas mass. Therefore, it is possible that dynamical hardening is important for some subsection of the population here, particularly at small separations. This implies that there could be complicated dynamics at work (including exchange encounters, binary hardening, or ionization). In particular, some of the lighter BH-BH binaries could exchange partners or be ionized in such encounters, and that might change the results in this paper.

The finding in Ref. [26] showed about $80-90 \%$ of mergers occur away from migration traps, and $10-20 \%$ of mergers occur at traps. This means that the migration time may be 
weakly correlated with the mergers because most of mergers occur within the migration time. That provides a strong argument for our neglect of the migration time.

\section{AGN disk}

We consider a geometrically thin, optically thick, radiatively efficient, and steady-state accretion disk [56], which is expected in AGNs [57]. We assume the viscosity parameter $\alpha=0.3$ [58]. We adopt radiation efficiency $\epsilon=$ $L_{\bullet \text {,Edd }} / \dot{M}_{\bullet \text {,Edd }} c^{2}=0.1$, where $L_{\bullet \text {,Edd }}$ and $\dot{M}_{\bullet \text {,Edd }}$ are the Eddington luminosity and the corresponding accretion rate of the $\mathrm{SMBH}$, respectively. Generally, the dimensionless accretion rate $\dot{m}_{\bullet}=\dot{M}_{\bullet} / \dot{M}_{\bullet}$,Edd $=0.1$ is adopted [57]. With the given $\alpha, \epsilon$, and $\dot{m}_{\bullet}$, one can estimate the surface density $\Sigma(r)$, the scale height $H(r)$, and the isothermal sound speed $c_{\mathrm{s}}(r)$ of the accretion disk around the SMBH based on the Eqs. (3) - (13) of Ref. [59].

It should be noted that the Eq. (3) of Ref. [59] is valid only with $Q \equiv c_{\mathrm{s}} \Omega /(\pi G \Sigma) \gtrsim 1$, where $\Omega=\left(G M_{\bullet} / r^{3}\right)^{1 / 2}$ is the Keplerian angular velocity of the disk. If not, the Eq. (3) should be replaced by the Eq. (15) in Ref. [59]. In addition, the viscosity is assumed to be proportional to total pressure of the gas, and the opacity is calculated based on the Eq. (10) of Ref. [33] (see also Fig. 1 of Ref. [60]). The nature of BH-disk interactions has large uncertainties in inhomogeneous (clumpy) disk beyond a radii $R_{\text {disk }}=0.1\left(M_{\bullet} / 10^{6} M_{\odot}\right)^{1 / 2} \mathrm{pc}$, where the AGN disk becomes the self-gravitating with the Toomre Qparameter, $Q \sim 1$ [61]. Therefore, we conservatively neglect the BH-disk interactions in this inhomogeneous (clumpy) region of the disk.

\section{Monte Carlo simulations}

We divide the AGN lifetime into five epochs, i.e., $\left\{\Delta t_{1} \in\right.$ $(0,2], \Delta t_{2} \in(2,4], \Delta t_{3} \in(4,6], \Delta t_{4} \in(6,8], \Delta t_{5} \in$ $(8,10]\}$ Myr. Here, a fiducial lifetime of $\tau_{\mathrm{AGN}}=10 \mathrm{Myr}$ is adopted for the AGN $[25,33,46]$. We assume the formation and mergers will occur by interaction between the initial single BHs aligning with the AGN disk within the first epoch. We further assume the initial binary BHs aligning with the disk within the epoch will participate in mergers but not interact with the other BHs in the disk to form binaries until the next epoch. Then, the BHs include two parts in the next epoch, that one is the initial single/binary BHs aligning with the disk in the next epoch and the other is the remnant BHs coming from the last epoch. We repeat the above method to calculate mergers in each epoch.

We assume that the binaries are randomly formed within the AGN disk in a certain fraction $f_{\text {bin }}$ which is the fraction of $\mathrm{BHs}$ in forming $\mathrm{BH}-\mathrm{BH}$ binaries in the AGN lifetime. Reference [36] considered the lower limit 0.01 and the upper limit 0.2 for the formation fraction. But Ref. [42] showed a larger fraction $\sim 0.6-0.8$ in their simulations. Reference [52] also found the average number of mergers per $\mathrm{BH}$ is about 0.4 within 30
Myr. Therefore, we adopt an appropriate formation fraction with $f_{\text {bin }}=0.5$ in our simulations.

In our simulations, we assume no binary in the AGN disk is disrupted via so called ionization due to the much shorter merger time than the ionization time [24, 62]. For simplicity, we neglect the increase in mass due to accretion and mass loss via mergers. We also neglect the merger kick received by the merger remnant because of larger orbital velocities $\sim 2 \times 10^{4} \mathrm{~km} / \mathrm{s}$. There are four distinct populations of BHs aligning with the disk as follows: $\left[a^{+}, L^{+}\right],\left[a^{+}, L^{-}\right],\left[a^{-}, L^{+}\right]$ and $\left[a^{-}, L^{-}\right]$, where $a^{+}\left(a^{-}\right)$and $L^{+}\left(L^{-}\right)$represent prograde (reprograde) spins and prograde (reprograde) orbits, respectively $[36,63]$. Here we mainly concentrate on mass distributions in mergers, and assume the masses of $\mathrm{BBH}$ mergers are unaffected on BH spins and orbits.

\section{RESULTS}

\section{A. BH populations in AGN disks}

In the AGN lifetime, we find the fraction $\sim 5.5 \%$ of the BHs align with the AGN disk, in which the fraction of the single BHs is $\sim 5.2 \%$ and the fraction of the BHs in binary is $\sim 7.2 \%$. The reason of the different fractions from the single/binary $\mathrm{BHs}$ is because we simulate an initial $\mathrm{BBH}$ as a single $\mathrm{BH}$ by using its total mass to calculate the alignment time [24], while Ref. [33] found heavier BHs align with the disk more quickly. Our fraction of BHs aligning with the AGN disk is consistent of Ref. [24]. However, we find the number of the BHs aligning the disk is different over time in the AGN lifetime. In our simulations, the $\{43.7 \%, 18.6 \%, 14.4 \%, 12.3 \%, 11.0 \%\}$ of $\mathrm{BHs}$ align with the disk within each epoch $\left\{\Delta t_{1}, \Delta t_{2}, \Delta t_{3}, \Delta t_{4}, \Delta t_{5}\right\}$. Therefore, it is necessary to calculate mergers as a function of time due to the differences of mergers in masses and numbers. We consider an epoch with $2 \mathrm{Myr}$, in which could assure that there is enough time to interact between most BHs in the AGN disk to form binaries and merge, but very few BHs have repeat mergers. For comparison, we also divide the AGN lifetime into four or six epochs and find that our results are robust. The process (see Sec. II) can naturally comprise a hierarchical $\mathrm{BBH}$ merger population to explore the mergers which have the extreme mass ratio or heavy component masses.

We define that a first generation, or $1 \mathrm{~g}$, merger, namely a primary merger, is the merger of two BHs that each came from the initial BH population. The merger will be hierarchical if either of the two BHs is the remnant of a pervious merger. It is worthwhile to be noted that the previous study (e.g., Ref. [46]) only considered a hierarchical merger in which at least one of the BHs came from the initial $\mathrm{BH}$ population. Here, we suggest that a merger of two BHs that one is the remnant of a $n$-g merger and the other is the remnant of a $m$-g merger, is referred to as a $(n+m+1)$-g merger. If the value of $n$ (or $m)$ is 0 , the remnant of the $n$ (or $m$ )-g merger represents a $\mathrm{BH}$ which came from the initial $\mathrm{BH}$ population. It is noted that the interpretation of generation is extended here. Because, strictly, a merger of two BHs that one is the remnant of a $n$-g merger 
TABLE I. The fractions of the mergers for different epochs and generations

\begin{tabular}{lcccccc}
\hline \hline & \multicolumn{6}{c}{ Fraction } \\
\cline { 2 - 7 } & \multicolumn{6}{c}{ Epoch } \\
\cline { 2 - 7 } Generation & $\Delta t_{1}$ & $\Delta t_{2}$ & $\Delta t_{3}$ & $\Delta t_{4}$ & $\Delta t_{5}$ & All \\
\hline $1 \mathrm{~g}$ & $21.61 \%$ & $15.00 \%$ & $14.07 \%$ & $13.22 \%$ & $12.24 \%$ & $76.13 \%$ \\
$2 \mathrm{~g}$ & 0 & $2.93 \%$ & $4.20 \%$ & $5.01 \%$ & $5.72 \%$ & $17.86 \%$ \\
$3 \mathrm{~g}$ & 0 & $0.23 \%$ & $0.83 \%$ & $1.47 \%$ & $2.09 \%$ & $4.62 \%$ \\
$4 \mathrm{~g}$ & 0 & 0 & $0.11 \%$ & $0.33 \%$ & $0.64 \%$ & $1.08 \%$ \\
$\geq 5 \mathrm{~g}$ & 0 & 0 & $0.01 \%$ & $0.07 \%$ & $0.22 \%$ & $0.31 \%$ \\
All & $21.61 \%$ & $18.16 \%$ & $19.22 \%$ & $20.10 \%$ & $20.91 \%$ & $100.00 \%$ \\
\hline \hline
\end{tabular}

and the other is the remnant of a $\mathrm{m}$-g merger, is referred to as a $(\max \{n, m\}+1)$-g merger. However, if that, properties of mergers between generations may be indistinct.

In Table I, we show the merger fractions in each epoch and each generation. We find the fractions $\sim 76 \%$ and $24 \%$ of the primary mergers and the hierarchical mergers, respectively, that are consistent of the result of Ref. [26] in bulk mergers and Ref. [52] combining N-body simulations. Moreover, Ref. [46] assumed that the fractions of each generation obey a Poisson distribution and found that the fraction of hierarchical mergers is up to $\sim 50 \%$. Those suggest that AGN disks are promising sites for the formation and mergers of hierarchical binary BHs. We see that the fraction of hierarchical mergers increases over time, particularly among which the fraction of $2 \mathrm{~g}$ mergers can go up to $\sim 6 \%$ in the late stage of the AGN. In contrast, the fraction of primary mergers reduces over time, but it still dominates the mergers during the AGN lifetime. In hierarchical mergers, $2 \mathrm{~g}$ mergers are in the majority and its fraction can reach up to $\sim 18 \%$ in the AGN lifetime. However, the high-generation mergers are smaller than the low-generation mergers, even exceedingly rare for fifthor above-generation mergers. In addition, Table I reveals that the fractions of BBH mergers in each epoch are with no difference. This indicates that the $\mathrm{AGN}$-assisted $\mathrm{BBH}$ merger rate is time-independent in the lifetime of the AGN in our simulations.

\section{B. Mass distribution of time-dependent mergers}

In Fig. 1, we show the primary mass $\left(m_{1}\right)$ distribution of mergers in each epoch. We see that the primary mass distribution increases in the late stage of the AGN due to the number of hierarchical mergers increases over time. Therefore, the detection rate of LIGO/Virgo will increase in the late stage of the AGN because of an invariant merger rate in AGN lifetime but the mass of mergers becomes heavier over time. This suggests LIGO/Virgo will detect a greater number of mergers in the late stage of the AGN, in which the fraction of hierarchical mergers will be expected to exceed $40 \%$, even up to $65 \%$ because of the heavier $\mathrm{BH}$ masses existing in hierarchical mergers. To verify the increase of the detection rate, we also plot the chirp mass $\left(\mathcal{M}=\left(m_{1} m_{2}\right)^{3 / 5} /\left(m_{1}+m_{2}\right)^{1 / 5}\right)$ distribution in each epoch

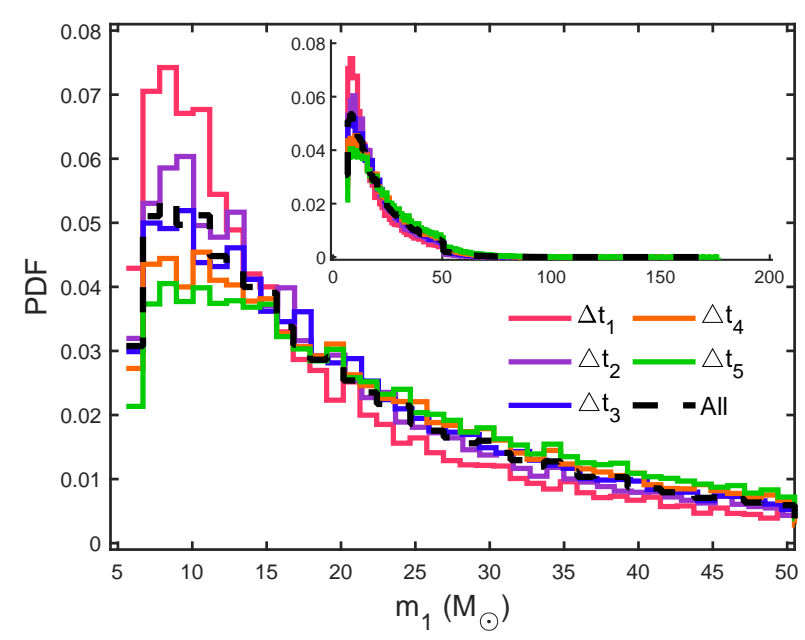

FIG. 1. The probability density distribution function of primary masses $\left(m_{1}\right)$ in mergers for different epochs, and the distribution function with $m_{1}$ of all epochs combined. The main figure shows the distribution function of $m_{1}$ in the range from $5 M_{\odot}$ to $50 M_{\odot}$, while the inset shows the distribution function for a wider range of masses. We show that the epochs $\Delta t_{1} \in(0,2] \mathrm{Myr}$ (red solid line), $\Delta t_{2} \in(2,4] \operatorname{Myr}$ (purple solid line), $\Delta t_{3} \in(4,6] \mathrm{Myr}$ (blue solid line), $\Delta t_{4} \in(6,8]$ Myr (orange solid line), $\Delta t_{5} \in(8,10]$ Myr (green solid line), and all epochs with the black dash line.

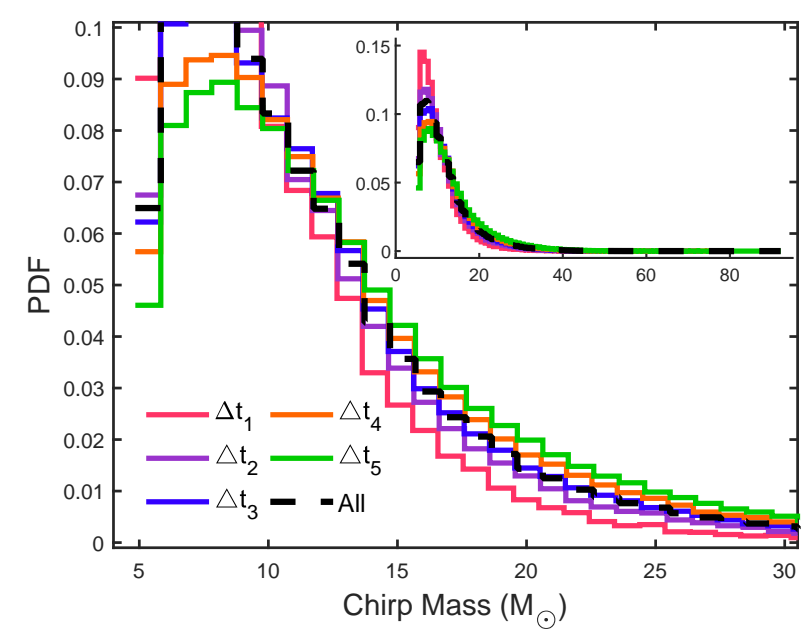

FIG. 2. The chirp mass $(\mathcal{M})$ distribution function of the mergers in each epoch. The legend is the same as Fig. 1.

in Fig. 2.

We derive the distribution function of the binaries' mass ratios $\left(q \equiv m_{2} / m_{1}\right)$ for the mergers in different epochs and show it in Fig. 3, where $m_{2}$ is the second mass of a BBH and lighter than the primary mass $m_{1}$. We find the mass ratio distribution of the epoch $\Delta t_{1}$ is roughly monotonous and reach the maximum in $q \sim 1$ but the others have peaks in $q \sim 0.2-0.5$. This indicates that LIGO/Virgo will detect mergers with a extreme mass ratio in the late stage of the AGN. That may be a possibility for gravitational wave binaries detected by 


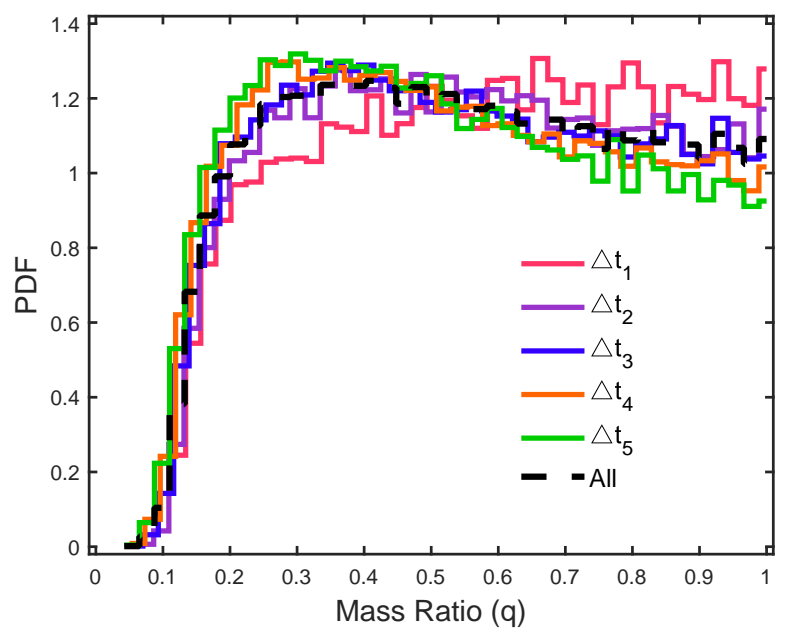

FIG. 3. The probability density distribution function of mass ratios of BBHs in mergers, we take account the contribution from each epoch. The legend is the same as Fig. 1.

LIGO/Virgo with the extreme mass ratio or heavy component masses, such as GW170729 [64], GW190412 [65], or even GW190426_190642 with total mass $\sim 184 M_{\odot}$ reported in GWTC-2.1 [66]. In addition, since Table I shows that the fraction of primary mergers still is larger compared to that of hierarchical mergers for each epoch in the AGN lifetime, the change of the primary mass distribution is not seemingly obvious from the inset of Fig. 1. However, it should be noted that the change in the trend will be magnified by LIGO/Virgo due to extreme mass-ratio mergers in the late stage of the AGN.

\section{Mass distribution of hierarchical mergers}

In Fig. 4, we show the primary mass distribution for different generation mergers. We see that the primary mass distribution increases significantly in high-generation mergers as expected, that is same as the distribution for different epochs. Reference [46] showed that a fraction $\sim 30 \%$ of mergers will have a BH with mass higher than the $50 M_{\odot}$ upper limit expected from stellar evolution [67-69]. However, our results cannot reach the high percentage because of about $24 \%$ of hierarchical mergers is obtained in our simulations but about $50 \%$ is found in their results.

We calculate the probability density distribution of mass ratios of the binaries for different generation mergers and show it in Fig. 5. We see that the mass ratio of the hierarchical mergers has the peak in the range about from 0.15 to 0.35 , in which the peak of $4 \mathrm{~g}$ mergers can reach to $\sim 0.15$. Reference [70] predicted that $99 \%$ of events should have $q \gtrsim 0.5$ via the $\mathrm{BH}$ population detected during the first and second observing run of Advanced LIGO (O1 and O2). However, we find that about $45 \%$ of mergers can have $q<0.5$ in the AGN disk, and it can rise up to $60 \%$ for the hierarchical mergers. Furthermore, it is worth to be noted that the mass ratio of $\geq 5 \mathrm{~g}$ mergers distinctly has an approximate flat distribution around its peak, and the

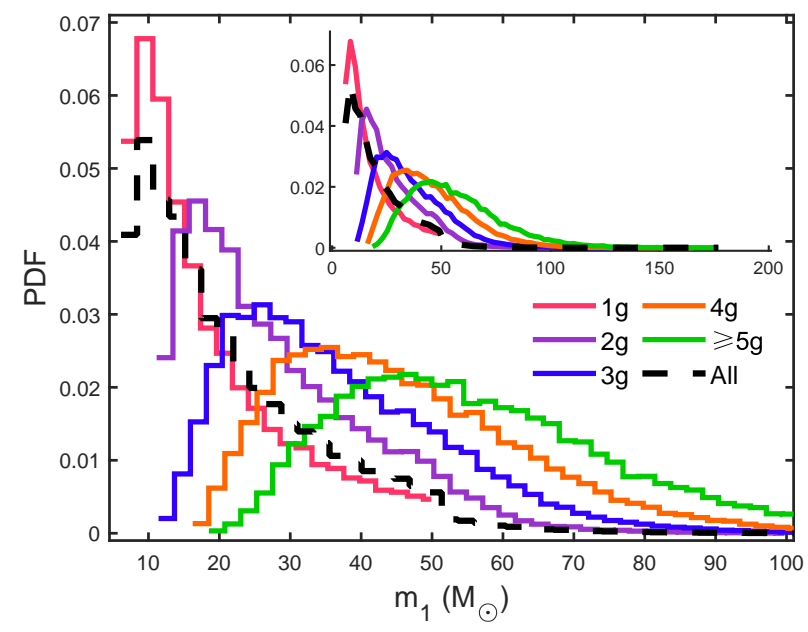

FIG. 4. The probability density distribution function of primary masses $\left(m_{1}\right)$ for different generation mergers, and the distribution function with $m_{1}$ of all generations combined. The main figure shows the distribution function of $m_{1}$ in the range from $5 M_{\odot}$ to $100 M_{\odot}$, while the inset shows the distribution function for a wider range of masses. We show that $1 \mathrm{~g}$ mergers (red solid line), $2 \mathrm{~g}$ mergers (purple solid line), $3 \mathrm{~g}$ mergers (blue solid line), $4 \mathrm{~g}$ mergers (orange solid line), $5 \mathrm{~g}$ and above $5 \mathrm{~g}$ mergers (green solid line), and all mergers with the black dash line.

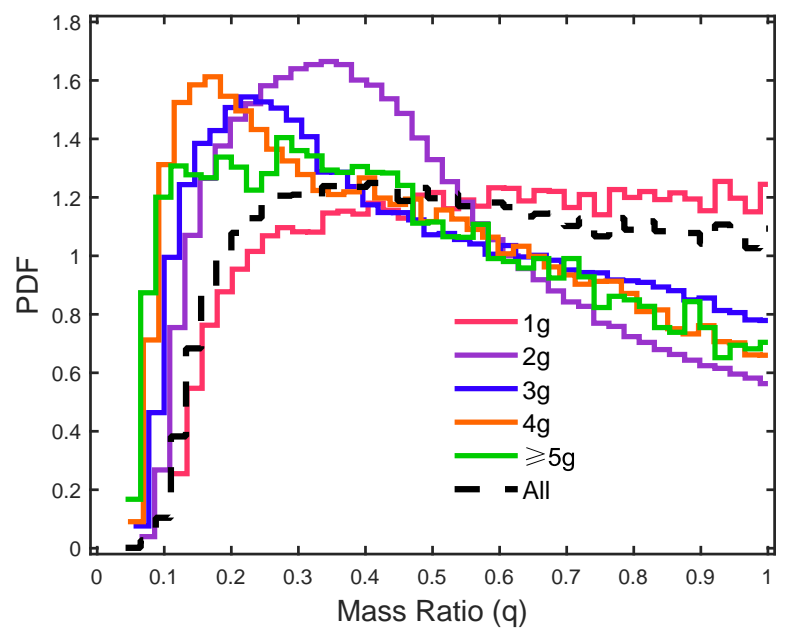

FIG. 5. The probability density distribution function of mass ratios of BBHs in mergers, we take account the contribution from each generation. The legend is the same as Fig. 4.

value of the peak is also smaller than other hierarchical mergers. Because we calculate hierarchical mergers including the mergers which are the results of merging from two BHs that at least each came from the remnant of a $1 \mathrm{~g}$ merger. That will induce the decline of the value of $q$ around its peak in highgeneration mergers. This also is a reason that the value of the peak is larger in second-generation mergers but not thirdor above-generation mergers. Therefore, we suggest that the mass ratio of high-generation mergers has a flatter distribution 

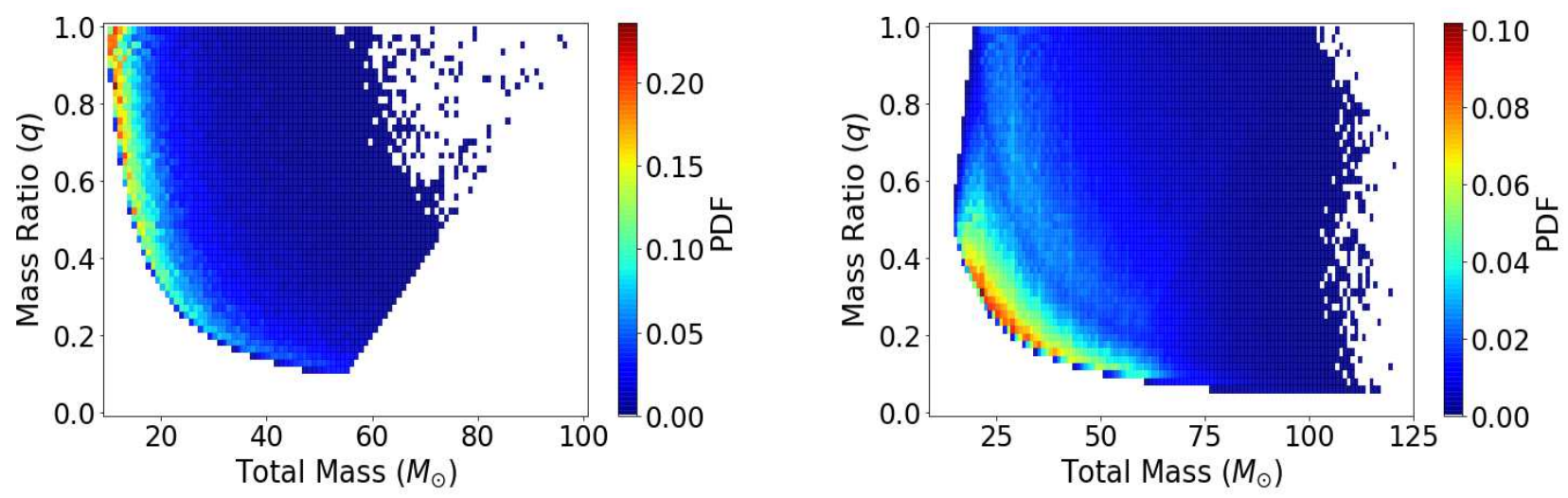

FIG. 6. 2D probability densities of the total mass and the mass ratio for the primary (left) and hierarchical (right) mergers.

around its peak than low-generation mergers in hierarchical mergers. Anyway, the minimum of $q \sim 0.03$ still comes from highest-generation mergers, i.e., $\geq 5 \mathrm{~g}$ mergers. The extreme unequal masses of mergers detected by LIGO/Virgo will be well explained if these occurred in AGN disks. We also show 2D probability densities of the total mass and mass ratio for mergers in the AGN disk in Fig. 6. In the left panel, we plot mergers only including the primary mergers, and in the right is the hierarchical mergers.

\section{Merger rate}

Reference [36] parameterized the rate of BH-BH mergers in AGN disks simply as:

$$
\begin{aligned}
R= & 12 \mathrm{Gpc}^{-3} \mathrm{yr}^{-1} \frac{N_{\mathrm{GN}}}{0.006 \mathrm{Mpc}^{-3}} \frac{N_{\mathrm{BH}}}{2 \times 10^{4}} \frac{f_{\mathrm{AGN}}}{0.1} \\
& \times \frac{f_{\mathrm{d}}}{0.1} \frac{f_{\text {bin }}}{0.1} \frac{\epsilon}{1}\left(\frac{\tau_{\mathrm{AGN}}}{10 \mathrm{Myr}}\right)^{-1},
\end{aligned}
$$

where $N_{\mathrm{GN}}$ is the average number density of galactic nuclei in the universe, $N_{\mathrm{BH}}$ is the number of BHs in an AGN disk, $f_{\mathrm{AGN}}$ is the fraction of galactic nuclei that have active AGNs that last for time $\tau_{\mathrm{AGN}}, f_{\mathrm{d}}$ is the fraction of BHs that end up in the AGN disk, and $\epsilon$ represents the fractional change in $N_{\mathrm{BH}}$ over one full AGN duty cycle. Using our finding that, within $1.2 \mathrm{pc}$ of an AGN disk 5.5\% of BHs end up in the disk, one can give a merger rate of $33 \mathrm{Gpc}^{-3} \mathrm{yr}^{-1}$.

We noticed that the maximal semimajor axis $R_{\text {inf }}$ of the AGN disk used here is $1.2 \mathrm{pc}$, which may be very large $O\left(10^{7}\right) r_{\mathrm{g}}$ for a $10^{6} M_{\odot} \mathrm{SMBH}$. Even if we are only considering $\mathrm{BH}$ migration within $0.1 \mathrm{pc}$ due to $Q<1$ outside this radii, that is $O\left(10^{6}\right) r_{\mathrm{g}}$ which is still very large. Note that migration time which can be short in the inner disk depends on the semimajor axis and surface density of the disk. Type I migration timescale will be very long in the outskirts of the disk model, i.e., in a Ref. [59] model disk type I migration timescale is $>10 \mathrm{Myr}$ for objects of $10 M_{\odot}$ at the radii of the disk $>10^{4} r_{\mathrm{g}}$. Most of the initially embedded population will be at large radii and not make it into the inner disk (near migration traps). However, any mergers that occur far out in the inner disk require very efficient gas hardening, or a random initially hard binary.

Therefore, we check how the rate of mergers change by making the AGN disk radii to $10^{5} r_{\mathrm{g}}$ or $10^{4} r_{\mathrm{g}}$. We find that within $10^{5} r_{\mathrm{g}}$ and $10^{4} r_{\mathrm{g}}$ of an AGN disk $\sim 6.2 \%$ and $4.5 \%$ of BHs align with the disk in the AGN lifetime, respectively. The difference in the fractions depends largely on the surface density of the disk which has the maximum between $10^{4} r_{\mathrm{g}}$ and $10^{5} r_{\mathrm{g}}\left(\tau_{\text {align }} \propto \Sigma^{-1}\right)$. Based on these, we find the allowed range of the BBH merger rate is $R \sim 27-37 \mathrm{Gpc}^{-3} \mathrm{yr}^{-1}$.

\section{CONCLUSIONS}

In this paper, we examine mass distributions of BBH mergers in AGN disks and consider the differences in the timedependent mergers and hierarchical mergers. Note that according to Eq. (6), in fact we neglect the change of radii of BHs when these align with the disk (i.e., $\mathrm{d} a / \mathrm{d} t \approx 0$ per orbit) and only concentrate on the timescale of orbital alignment. Reference [42] found that the last $\mathrm{BHs}$ to reach the migration trap region are the innermost $\mathrm{BHs}$ which have a large initial semimajor axes and small inclination angle [71]. Therefore, time (not migration time) from BHs aligning the disk to formation (mergers) of binaries is strongly dependent on radii of BHs when these align with the disk. This may largely impact on the binaries effective spin $\left(\chi_{\text {eff }}=\left(m_{1} \chi_{1} \cos \theta_{1}+\right.\right.$ $\left.m_{2} \chi_{2} \cos \theta_{2}\right) /\left(m_{1}+m_{2}\right)$, where $\chi_{i}$ and $m_{i}$ are the spin and mass, respectively, of each $\mathrm{BH}$ in the merged $\mathrm{BBH}$, and the spin obliquity $\theta_{i}$ is the angle between the spin $\chi_{i}$ vector and the orbital angular momentum vector) but not mass and mass ratio because we do not consider the migration time which is much shorter than the alignment time.

For $\chi_{\text {eff }}$, it should have a large value by merging due to gas accretion from the AGN disk that will tend to torque the $\mathrm{BH}$ spin into alignment with the gas after about $1-10 \%$ of the gas has been accreted (e.g., Ref. [72]). After $10 \mathrm{Myr}$ any BHs that have been present in the disk should have been torqued into alignment with the disk orbital angular momentum. Therefore, the population of mergers in the late AGN disk (and associated mass and mass ratio distributions) should therefore 
be biased towards large values of $\chi_{\text {eff }}$. Early populations are more likely to have random spin alignments and therefore a range of $\chi_{\text {eff }}$ centered around zero. So, by testing the population of early and late AGN disks, we also could probe the likely torquing by disk accretion onto the embedded objects.

Our conclusions are the following:

(1) The BBH merger rate which is time-independent (see Table I) is $\sim 27-37 \mathrm{Gpc}^{-3} \mathrm{yr}^{-1}$ in the AGN lifetime. However, the detection rate of LIGO/Virgo accompanying with extreme mass-ratio mergers (see Fig. 3) will increase over time (see Fig. 2) due to heavy component masses (see Fig. 1) in the AGN lifetime.

(2) The fraction of the hierarchical mergers is $\sim 24 \%$, in which $2 \mathrm{~g}$ mergers dominate with $\sim 18 \%$ (see Table I).

(3) The peak of the mass ratio of hierarchical mergers is $q \sim 0.15-0.35$, in which $4 \mathrm{~g}$ can reach to $\sim 0.15$ (see Fig. 5 , also Fig. 6).

(4) The mass ratio of the high-generation mergers has an approximate flatter distribution around its peak than the lowgeneration mergers (see Fig. 5), and 2g mergers have larger value of the peak in mass ratios. Because a hierarchical merger of two BHs that each possibly came from the remnant of a previous merger. Such consideration is different from Ref. [46].

(5) About $45 \%$ of $\mathrm{BBH}$ mergers in AGN disks have mass ratio $q<0.5$ (see Fig. 3), while it can go up to $\sim 60 \%$ for hierarchical mergers (see Fig. 5).

(6) If the BBH mergers detected by LIGO/Virgo with the extreme mass ratio or heavy component masses can be well explained in the AGN channel.

\section{ACKNOWLEDGMENTS}

We thank the anonymous referee for beneficial suggestions that improved the paper. We are grateful to Da-Bin Lin for useful suggestions. This work is supported by the National Natural Science Foundation of China (Grant No. 11773007) and the Guangxi Science Foundation (Grant No. 2018GXNSFFA281010).
[1] J. Aasi et al., "Characterization of the LIGO detectors during their sixth science run," Classical and Quantum Gravity 32, 115012 (2015), arXiv: 1410.7764 .

[2] F. Acernese et al., "Advanced Virgo: a secondgeneration interferometric gravitational wave detector, $\quad$ Classical and Quantum Gravity 32, 024001 (2015), arXiv:1408.3978.

[3] A. Tutukov and L. Yungelson, "Evolution of massive close binaries," Nauchnye Informatsii 27, 70 (1973).

[4] M. Dominik, K. Belczynski, C. Fryer, D. E. Holz, E. Berti, T. Bulik, I. Mandel, and R. O'Shaughnessy, "Double Compact Objects. I. The Significance of the Common Envelope on Merger Rates,” Astrophys. J. 759, 52 (2012), arXiv:1202.4901.

[5] M. Spera, M. Mapelli, N. Giacobbo, A. A. Trani, A. Bressan, and G. Costa, "Merging black hole binaries with the SEVN code," Mon. Not. R. Astron. Soc. 485, 889 (2019), arXiv: 1809.04605.

[6] L. A. C. van Son, S. E. de Mink, T. Callister, S. Justham, M. Renzo, T. Wagg, F. S. Broekgaarden, F. Kummer, R. Pakmor, and I. Mandel, "The redshift evolution of the binary black hole merger rate: a weighty matter," arXiv e-prints , arXiv:2110.01634 (2021), arXiv:2110.01634.

[7] E. P. J. van den Heuvel, S. F. Portegies Zwart, and S. E. de Mink, "Forming short-period Wolf-Rayet Xray binaries and double black holes through stable mass transfer," Mon. Not. R. Astron. Soc. 471, 4256 (2017), arXiv:1701.02355.

[8] Y. Shao and X.-D. Li, "Population Synthesis of Black Hole Binaries with Compact Star Companions," arXiv e-prints , arXiv:2107.03565 (2021), arXiv:2107.03565.

[9] A. Olejak, K. Belczynski, and N. Ivanova, "Impact of common envelope development criteria on the formation of LIGO/Virgo sources," Astron. Astrophys 651, A100 (2021), arXiv:2102.05649.

[10] H. Tagawa, T. R. Saitoh, and B. Kocsis, "Compact Object Mergers Driven by Gas Fallback,"
Phys. Rev. Lett. 120, 261101 (2018), arXiv:1802.00441.

[11] S. E. de Mink and I. Mandel, "The chemically homogeneous evolutionary channel for binary black hole mergers: rates and properties of gravitational-wave events detectable by advanced LIGO,” Mon. Not. R. Astron. Soc. 460, 3545 (2016), arXiv:1603.02291.

[12] P. Marchant, N. Langer, P. Podsiadlowski, T. M. Tauris, and T. J. Moriya, "A new route towards merging massive black holes," Astron. Astrophys 588, A50 (2016), arXiv:1601.03718.

[13] M. Mapelli, Y. Bouffanais, F. Santoliquido, M. Arca Sedda, and M. C. Artale, "The Cosmic Evolution of Binary Black Holes in Young, Globular and Nuclear Star Clusters: Rates, Masses, Spins and Mixing Fractions," arXiv e-prints , arXiv:2109.06222 (2021), arXiv:2109.06222.

[14] S. Banerjee, "Stellar-mass black holes in young massive and open stellar clusters and their role in gravitationalwave generation,” Mon. Not. R. Astron. Soc. 467, 524 (2017), arXiv:1611.09357.

[15] S. Rastello, P. Amaro-Seoane, M. Arca-Sedda, R. Capuzzo-Dolcetta, G. Fragione, and I. Tosta e Melo, "Stellar black hole binary mergers in open clusters," $\quad$ Mon. Not. R. Astron. Soc. 483, 1233 (2019), arXiv:1811.10628.

[16] J. Kumamoto, M. S. Fujii, and A. Tanikawa, "Merger rate density of binary black holes formed in open clusters," Mon. Not. R. Astron. Soc. 495, 4268 (2020), arXiv:2001.10690.

[17] S. F. Portegies Zwart and S. L. W. McMillan, "Black Hole Mergers in the Universe," Astrophys. J. 528, L17 (2000), arXiv:astro-ph/9910061.

[18] A. W. H. Kamlah et al., "Preparing the next gravitational million-body simulations: Evolution of single and binary stars in Nbody6++GPU, MOCCA and McLuster," arXiv e-prints , arXiv:2105.08067 (2021), arXiv:2105.08067.

[19] F. Antonini and F. A. Rasio, "Merging Black Hole Binaries in Galactic Nuclei: Implications for Advanced-LIGO Detections," Astrophys. J. 831, 187 (2016), arXiv:1606.04889. 
[20] M. Zevin, J. Samsing, C. Rodriguez, C.-J. Haster, and E. Ramirez-Ruiz, "Eccentric Black Hole Mergers in Dense Star Clusters: The Role of Binary-Binary Encounters," Astrophys. J. 871, 91 (2019), arXiv:1810.00901.

[21] F. Zhang, L. Shao, and W. Zhu, "Gravitational-wave Merging Events from the Dynamics of Stellar-mass Binary Black Holes around the Massive Black Hole in a Galactic Nucleus," Astrophys. J. 877, 87 (2019), arXiv:1903.02685.

[22] G. Fragione, A. Loeb, and F. A. Rasio, "On the Origin of GW190521-like Events from Repeated Black Hole Mergers in Star Clusters," Astrophys. J. 902, L26 (2020), arXiv:2009.05065.

[23] B. McKernan, K. E. S. Ford, W. Lyra, and H. B. Perets, "Intermediate mass black holes in AGN discs - I. Production and growth," Mon. Not. R. Astron. Soc. 425, 460 (2012), arXiv:1206.2309.

[24] I. Bartos, B. Kocsis, Z. Haiman, and S. Márka, "Rapid and Bright Stellar-mass Binary Black Hole Mergers in Active Galactic Nuclei,” Astrophys. J. 835, 165 (2017), arXiv:1602.03831.

[25] Y. Yang, V. Gayathri, I. Bartos, Z. Haiman, M. Safarzadeh, and H. Tagawa, "Black Hole Formation in the Lower Mass Gap through Mergers and Accretion in AGN Disks," Astrophys. J. 901, L34 (2020), arXiv:2007.04781.

[26] B. McKernan, K. E. S. Ford, R. O'Shaugnessy, and D. Wysocki, "Monte Carlo simulations of black hole mergers in AGN discs: Low $\chi_{\text {eff }}$ mergers and predictions for LIGO," Mon. Not. R. Astron. Soc. 494, 1203 (2020), arXiv:1907.04356.

[27] H. Tagawa, Z. Haiman, I. Bartos, and B. Kocsis, "Spin Evolution of Stellar-mass Black Hole Binaries in Active Galactic Nuclei,” Astrophys. J. 899, 26 (2020), arXiv:2004.11914.

[28] B. McKernan, K. E. S. Ford, T. Callister, W. M. Farr, R. O'Shaughnessy, R. Smith, E. Thrane, and A. Vajpeyi, "LIGO-Virgo correlations between mass ratio and effective inspiral spin: testing the active galactic nuclei channel," arXiv e-prints , arXiv:2107.07551 (2021), arXiv:2107.07551.

[29] B. J. Carr and S. W. Hawking, "Black holes in the early Universe," Mon. Not. R. Astron. Soc. 168, 399 (1974).

[30] S. Wang and Z.-C. Zhao, "GW200105 and GW200115 are compatible with a scenario of primordial black hole binary coalescences," arXiv e-prints , arXiv:2107.00450 (2021), arXiv:2107.00450.

[31] F. Antonini, S. Toonen, and A. S. Hamers, "Binary Black Hole Mergers from Field Triples: Properties, Rates, and the Impact of Stellar Evolution,” Astrophys. J. 841, 77 (2017), arXiv:1703.06614.

[32] B. Liu and D. Lai, "Enhanced black hole mergers in binary-binary interactions," Mon. Not. R. Astron. Soc. 483, 4060 (2019), arXiv: 1809.07767.

[33] Y. Yang, I. Bartos, Z. Haiman, B. Kocsis, Z. Márka, N. C. Stone, and S. Márka, "AGN Disks Harden the Mass Distribution of Stellar-mass Binary Black Hole Mergers," Astrophys. J. 876, 122 (2019), arXiv:1903.01405.

[34] D. Gerosa and M. Fishbach, "Hierarchical mergers of stellarmass black holes and their gravitational-wave signatures," Nature Astronomy 5, 749 (2021), arXiv:2105.03439.

[35] J. M. Bellovary, M.-M. Mac Low, B. McKernan, and K. E. S. Ford, "Migration Traps in Disks around Supermassive Black Holes,” Astrophys. J. 819, L17 (2016), arXiv:1511.00005.

[36] B. McKernan et al., "Constraining Stellar-mass Black Hole Mergers in AGN Disks Detectable with LIGO," Astrophys. J. 866, 66 (2018), arXiv:1702.07818.
[37] J.-M. Wang, J.-R. Liu, L. C. Ho, and P. Du, "Accretionmodified Stars in Accretion Disks of Active Galactic Nuclei: Slowly Transient Appearance," Astrophys. J. 911, L14 (2021), arXiv:2103.07708.

[38] J.-M. Wang, J.-R. Liu, L. C. Ho, Y.-R. Li, and P. Du, "Accretion-modified Stars in Accretion Disks of Active Galactic Nuclei: Gravitational-wave Bursts and Electromagnetic Counterparts from Merging Stellar Black Hole Binaries," Astrophys. J. 916, L17 (2021), arXiv:2106.07334.

[39] B. McKernan, K. E. S. Ford, I. Bartos, M. J. Graham, W. Lyra, S. Marka, Z. Marka, N. P. Ross, D. Stern, and Y. Yang, "Ram-pressure Stripping of a Kicked Hill Sphere: Prompt Electromagnetic Emission from the Merger of Stellar Mass Black Holes in an AGN Accretion Disk," Astrophys. J. 884, L50 (2019), arXiv:1907.03746.

[40] J.-P. Zhu, K. Wang, B. Zhang, Y.-P. Yang, Y.-W. Yu, and H. Gao, "High-energy Neutrinos from Choked GammaRay Bursts in Active Galactic Nucleus Accretion Disks," Astrophys. J. 911, L19 (2021), arXiv:2103.00789.

[41] C. J. Walcher, R. P. van der Marel, D. McLaughlin, H. W. Rix, T. Böker, N. Häring, L. C. Ho, M. Sarzi, and J. C. Shields, "Masses of Star Clusters in the Nuclei of Bulgeless Spiral Galaxies,” Astrophys. J. 618, 237 (2005), arXiv:astro-ph/0409216.

[42] A. Secunda, J. Bellovary, M.-M. Mac Low, K. E. S. Ford, B. McKernan, N. W. C. Leigh, W. Lyra, and Z. Sándor, "Orbital Migration of Interacting Stellar Mass Black Holes in Disks around Supermassive Black Holes," Astrophys. J. 878, 85 (2019), arXiv:1807.02859.

[43] M. Morris, "Massive Star Formation near the Galactic Center and the Fate of the Stellar Remnants," Astrophys. J. 408, 496 (1993).

[44] C. J. Hailey, K. Mori, F. E. Bauer, M. E. Berkowitz, J. Hong, and B. J. Hord, "A density cusp of quiescent X-ray binaries in the central parsec of the Galaxy," Nature 556, 70 (2018).

[45] P. Peng and X. Chen, "The last migration trap of compact objects in AGN accretion disc," Mon. Not. R. Astron. Soc. 505, 1324 (2021), arXiv:2104.07685.

[46] Y. Yang et al., "Hierarchical Black Hole Mergers in Active Galactic Nuclei,” Phys. Rev. Lett. 123, 181101 (2019), arXiv: 1906.09281.

[47] J. N. Bahcall and R. A. Wolf, "The star distribution around a massive black hole in a globular cluster. II. Unequal star masses.” Astrophys. J. 216, 883 (1977).

[48] T. Panamarev, A. Just, R. Spurzem, P. Berczik, L. Wang, and M. Arca Sedda, "Direct N-body simulation of the Galactic centre,” Mon. Not. R. Astron. Soc. 484, 3279 (2019), arXiv:1805.02153.

[49] R. M. O'Leary, B. Kocsis, and A. Loeb, "Gravitational waves from scattering of stellar-mass black holes in galactic nuclei," Mon. Not. R. Astron. Soc. 395, 2127 (2009), arXiv:0807.2638.

[50] L. Gondán, B. Kocsis, P. Raffai, and Z. Frei, "Eccentric Black Hole Gravitational-wave Capture Sources in Galactic Nuclei: Distribution of Binary Parameters," Astrophys. J. 860, 5 (2018), arXiv:1711.09989.

[51] K. Gültekin et al., "The M- $\sigma$ and M-L Relations in Galactic Bulges, and Determinations of Their Intrinsic Scatter," Astrophys. J. 698, 198 (2009), arXiv:0903.4897.

[52] H. Tagawa, Z. Haiman, and B. Kocsis, "Formation and Evolution of Compact-object Binaries in AGN Disks," Astrophys. J. 898, 25 (2020), arXiv:1912.08218.

[53] A. Generozov, N. C. Stone, B. D. Metzger, and J. P. Ostriker, "An overabundance of black hole X- 
ray binaries in the Galactic Centre from tidal captures," Mon. Not. R. Astron. Soc. 478, 4030 (2018), arXiv:1804.01543.

[54] A. Secunda, J. Bellovary, M.-M. Mac Low, K. E. S. Ford, B. McKernan, N. W. C. Leigh, W. Lyra, Z. Sándor, and J. I. Adorno, "Orbital Migration of Interacting Stellar Mass Black Holes in Disks around Supermassive Black Holes. II. Spins and Incoming Objects,” Astrophys. J. 903, 133 (2020), arXiv:2004.11936.

[55] C. Baruteau, J. Cuadra, and D. N. C. Lin, "Binaries Migrating in a Gaseous Disk: Where are the Galactic Center Binaries?" Astrophys. J. 726, 28 (2011), arXiv:1011.0360.

[56] N. I. Shakura and R. A. Sunyaev, "Reprint of 1973A\&A....24..337S. Black holes in binary systems. Observational appearance." Astron. Astrophys 500, 33 (1973).

[57] B. Kocsis, N. Yunes, and A. Loeb, "Observable signatures of extreme mass-ratio inspiral black hole binaries embedded in thin accretion disks," Phys. Rev. D 84, 024032 (2011), arXiv:1104.2322.

[58] A. R. King, J. E. Pringle, and M. Livio, "Accretion disc viscosity: how big is alpha?" Mon. Not. R. Astron. Soc. 376, 1740 (2007), arXiv:astro-ph/0701803.

[59] E. Sirko and J. Goodman, "Spectral energy distributions of marginally self-gravitating quasi-stellar object discs," Mon. Not. R. Astron. Soc. 341, 501 (2003), arXiv:astro-ph/0209469.

[60] T. A. Thompson, E. Quataert, and N. Murray, "Radiation Pressure-supported Starburst Disks and Active Galactic Nucleus Fueling," Astrophys. J. 630, 167 (2005), arXiv:astro-ph/0503027.

[61] Z. Haiman, B. Kocsis, and K. Menou, "The Population of Viscosity- and Gravitational Wave-driven Supermassive Black Hole Binaries Among Luminous Active Galactic Nuclei," Astrophys. J. 700, 1952 (2009), arXiv:0904.1383.

[62] O. Pfuhl, T. Alexander, S. Gillessen, F. Martins, R. Genzel, F. Eisenhauer, T. K. Fritz, and T. Ott, "Massive Binaries in the Vicinity of Sgr A*," Astrophys. J. 782, 101 (2014),
arXiv:1307.7996.

[63] Y. Wang, B. McKernan, S. Ford, R. Perna, N. W. C. Leigh, and M.-M. Mac Low, "Symmetry Breaking in Dynamical Encounters in the Disks of Active Galactic Nuclei," arXiv e-prints , arXiv:2110.03698 (2021), arXiv:2110.03698.

[64] B. P. Abbott et al., "GWTC-1: A Gravitational-Wave Transient Catalog of Compact Binary Mergers Observed by LIGO and Virgo during the First and Second Observing Runs," Physical Review X 9, 031040 (2019), arXiv:1811.12907.

[65] R. Abbott et al., "GW190412: Observation of a binary-black-hole coalescence with asymmetric masses," Phys. Rev. D 102, 043015 (2020).

[66] The LIGO Scientific Collaboration et al., "GWTC-2.1: Deep Extended Catalog of Compact Binary Coalescences Observed by LIGO and Virgo During the First Half of the Third Observing Run,” arXiv e-prints , arXiv:2108.01045 (2021), arXiv:2108.01045.

[67] K. Belczynski et al., "The effect of pair-instability mass loss on black-hole mergers," Astron. Astrophys 594, A97 (2016), arXiv:1607.03116.

[68] S. E. Woosley, "Pulsational Pair-instability Supernovae," Astrophys. J. 836, 244 (2017), arXiv:1608.08939.

[69] N. Giacobbo and M. Mapelli, "The progenitors of compactobject binaries: impact of metallicity, common envelope and natal kicks," Mon. Not. R. Astron. Soc. 480, 2011 (2018), arXiv: 1806.00001.

[70] M. Fishbach, W. M. Farr, and D. E. Holz, "The Most Massive Binary Black Hole Detections and the Identification of Population Outliers,” Astrophys. J. 891, L31 (2020), arXiv:1911.05882.

[71] G. Fabj, S. S. Nasim, F. Caban, K. E. S. Ford, B. McKernan, and J. M. Bellovary, "Aligning nuclear cluster orbits with an active galactic nucleus accretion disc," Mon. Not. R. Astron. Soc. 499, 2608 (2020), arXiv:2006.11229.

[72] T. Bogdanović, C. S. Reynolds, and M. C. Miller, "Alignment of the Spins of Supermassive Black Holes Prior to Coalescence," Astrophys. J. 661, L147 (2007), arXiv:astro-ph/0703054. 